\title{
Radiation effect on shape memory alloy
}

Amine Riad ${ }^{\mathrm{a}, 1}$, Mouna Benzohra ${ }^{\mathrm{a}, 2}$, Mohamed Mansouri ${ }^{\mathrm{a}, 3}$ and Abdelilah ALHAMANY ${ }^{\mathrm{a}, 4}$ ${ }^{a}$ LMEET Laboratory, FST Settat, University Hassan $1^{\text {st }}$ city: Settat, country: Morocco.

Abstract. The shape memory alloys belong to the smart materials thanks to their thermomechanical proprieties' reply to thermal or to mechanical loading. These materials can change shape, stiffness, displacement, natural frequency, and many mechanical characteristics in response to stress or to heat such as conduction, convection or radiation. However, heating by convection or conduction are the most useful and studied methods unlike radiation. Therefore, this paper aims to study the radiation effect on the shape memory alloy behavior.

Keywords. Shape memory alloy, SMA, Smart material, Radiation effect, solar radiation effect, radiation heat.

\section{Introduction}

The Shape Memory Alloys (SMAs) are a smart material [1] that exhibit exceptional thermomecanical behavior in response to stress or to heat [2]. This is materials have many thermomechanical features as the shape memory effect (SME) and the superelasticity (SE)[3]. The superelasticity occurs when the material stressed at temperature above $A_{f}$, hence it is back to the original shape after the unloading. As well as the shape memory effect occurs when the material stressed at a defined temperature, hence it is back to the original shape after heating[4]. These particular characteristics have been observed in several alloys [5]; the most used in industrial field are the Ni-Ti and $\mathrm{Cu}-\mathrm{Al}-\mathrm{Be}$ alloys [6].

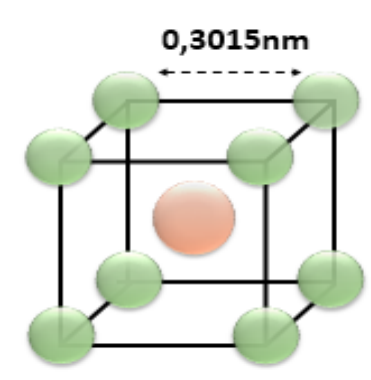

Austenite

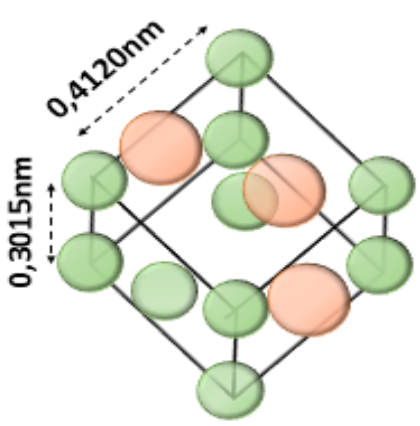

Martensite

Figure 1: Three-dimensional structure of austenite and martensite 
The martensitic transformation is the origin of all the amazing properties attributed to SMA it can be progressed either temperature stimulus or stress load [7]. The thermomechanical behavior of SMA [8] results from the interaction of two phases (figure 1): the first phase with cubic structure at higher temperature (austenite), the second phase with monoclinic structure at low temperature phase (martensite) [9]. In addition, the martensite phase can be exist as self-accommodated structure (low temperature, low stress state) or as stressinduced structure that can generate large strain [10].

At higher temperature $\left(A_{f}\right)$ the material is totally austenite and at lower temperature (Mf) the material is totally martensitic, $\left(\mathrm{M}_{\mathrm{s}}\right)$ and (As) are started temperature of the transformation phase for martensite and austenite respectively (figure 2).

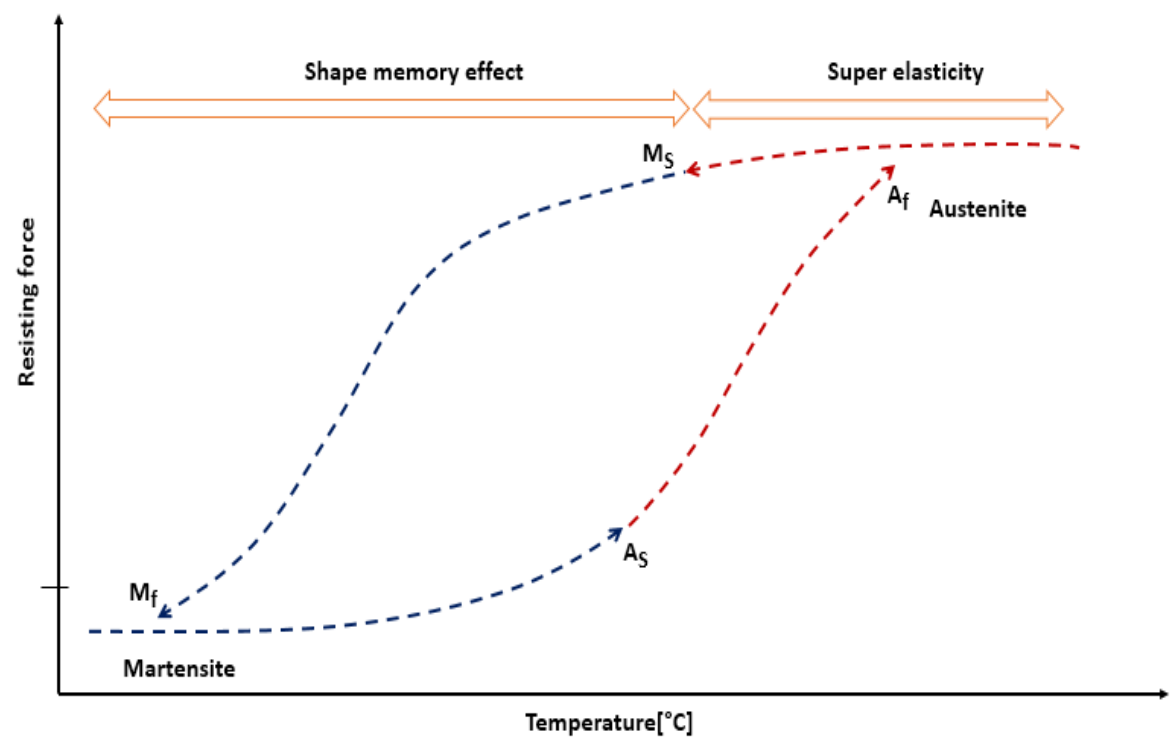

Figure 2: the martensitic transformation and principal thermomemechanical behaviour of shape memory alloy

The SMA reacts to heat that can be useful for sensors or actuators by producing mechanical energy, which can change shape, size or displacement [2] [10].

To some extent, the temperature of specific system can be increased by heat that can be transferred by three ways, which is convection, conduction or radiation, though, convection and conduction need matter to transfer heat [12]. However, radiation does not need intermediating to transfer because the thermal radiation is happened of random motions of the atoms. Therefore, the energy amount passed is determined by the temperature, and the nature of the surface emits the radiation. In addition, the heat energy transfers from hot system to cool system.

The radiation transfers as electromagnetic waves that can be transmitted by radio waves, gamma rays and others, no matter how the difference is only in the wavelength [13]. Once 
the rays encounter the surfaces, just a part of them can be passed through the surface when the other parts can be absorbed or reflected away. This depends on three radiative properties of the surface, which are $\rho$, reflectivity, $\alpha$, absorption and $\tau$, permeability. The sum of these three properties [14], can be described as:

$$
\rho+\alpha+\tau=1
$$

Indeed, the Black body is an imaginary perfect body that is the best absorber and emitter that can absorb all electromagnetic radiation. Since this body is ideal, then it can be releasing more energy better than anybody at similar temperature can.

Therefore, the SMAs can operate in several ways, such as conduction that expose to direct temperature or by electrical heating using the joule effect or radiat heat. At all events, the electrical heat and direct temperature are the most used methods for heating the shape memory alloys; unlike, the radiation effect is never used for heating.

In this paper, a numerical study aims to predict the shape memory alloy behavior reacts to radiation heat. In this way, an investigation intends to control the shape memory alloy using solar radiation to activate thermo-mechanical actuators that can be useful for multiple renewable energy applications.

\section{Modelling}

In this work, the thermo-mechanical model developed was adapted to capture the proprieties of the shape memory alloy, specially the shape memory effect and super elasticity. The fundamental conventions of this model are the elastic moduli that expected to be equal for austenite and martensitic.

In the frame of SMA thermomechanical behavior, the total strain, $\varepsilon_{\text {tot }}$, can be presented as the sum of partial strain adopted in the form [1]:

$$
\varepsilon_{\mathrm{tot}}=\varepsilon^{e l}+\varepsilon^{r a d}+\varepsilon^{T r}+\varepsilon^{p}
$$

The elastic strain, $\varepsilon_{\mathrm{e}}$ is:

$$
\varepsilon_{\mathrm{e}}=\sigma / \mathrm{E}
$$

The thermal radiation strain, $\varepsilon^{r a d}$ is:

$$
\varepsilon^{r a d}=\alpha \cdot \Delta T
$$


Consider $\alpha$, the coefficient of thermal expansion, $x$ the volume fraction, $\sigma$ denotes the applied stress, $\Delta \mathrm{T}$ is the temperature difference and $\mathrm{E}$ denotes the Young modulus.

The transformation strain, $\varepsilon^{T r}$ :

$$
\varepsilon^{T r}=\sum_{n=1}^{24} x^{n} R^{n}
$$

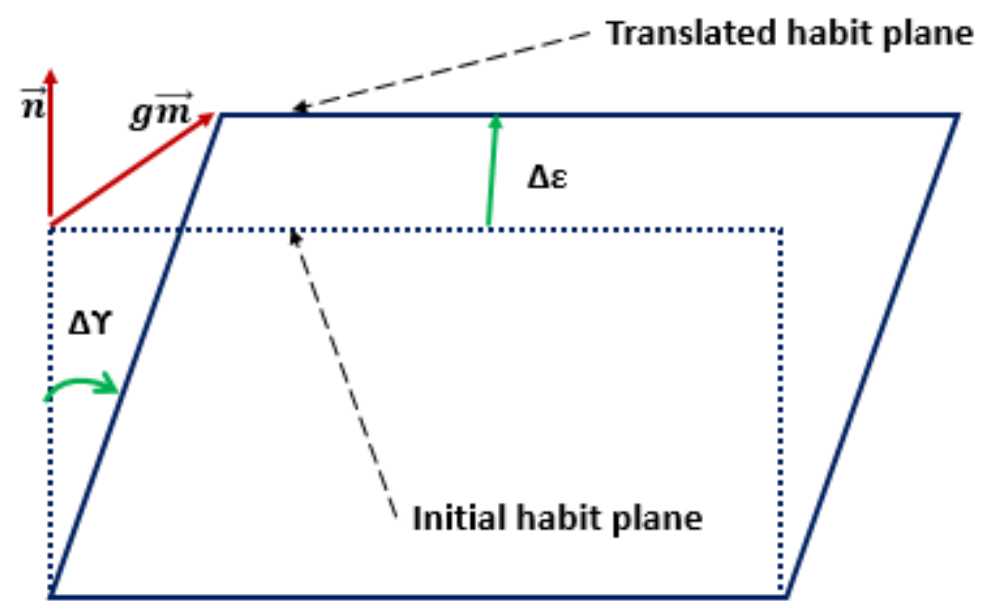

Figure 3: Martensitic transformation mechanism[15]

$$
R_{i j}^{n}=\frac{1}{2} g\left(n_{i}^{n} m_{j}^{n}+n_{j}^{n} m_{i}^{n}\right)
$$

$R$ is the orientation tensor, $g$ is the magnitude of shear deformation caused by the martensite transformation [16]

$$
\varepsilon^{P}=\sum_{n=1}^{24} \gamma^{n} R^{n}
$$

$\gamma^{n}$ is the slippage of the $\mathrm{n}$-th friction slip system.

The model uses the Helmholtz free energy for the derivation of constitutive relations, the constitutive functions are assumed that can be set-up as shown in equation (7):

$$
\begin{gathered}
\left.\Psi\left(T, \varepsilon^{e l}, \varepsilon^{t r}, \varepsilon^{p}, x,\right)=\frac{1}{2 \rho} \varepsilon^{e l}: E: \varepsilon^{e l}+C_{v}\left[\left(T-T_{0}\right)-T \ln \frac{T}{T_{0}}\right)\right]+\alpha\left(T-T_{0}\right) x+ \\
\int_{0}^{t} A \varepsilon^{p}: \dot{\varepsilon}^{t r} d t+\int_{0}^{t} \sum_{n=1}^{24} Y^{p} \dot{x}^{n} d t+\int_{0}^{t} \sum_{n=1}^{12} R^{n}\left|(1-x) \dot{\gamma}^{n}\right| d t
\end{gathered}
$$

$\int_{0}^{t} A \varepsilon^{p}: \dot{\varepsilon}^{t r} d t:$ is the interaction energy that reflects the effect of plasticity caused by the dislocation slip mechanism of the phase transformation.

$\int_{0}^{t} \sum_{n=1}^{24} Y^{p} \dot{x}^{n} d t$, defines the transformation hardening caused by the martensite transformation without the contribution of dislocation slip in the austenitic phase. 
$\int_{0}^{t} \sum_{n=1}^{12} R^{n}\left|(1-x) \dot{\gamma}^{n}\right| d t$ describes the work hardening caused by the sliding of dislocations in the austenitic phase.

Where $\gamma$ is the amount of irreversible friction slip, $\mathrm{Y}$ is the resistance to transformation, $\mathrm{E}$ is Young's modulus, $\mathrm{C}_{\mathrm{v}}$ : heat capacity, $\alpha$ is the thermal expansion coefficient, $\mathrm{T}_{0}$ is the equilibrium temperature, A The control parameter of the influence that facilitate or promote the sequential phase transformation.

$$
\Gamma=\sigma: \dot{\varepsilon}-\dot{\Psi}-\eta \dot{T}-\frac{Q . \nabla T}{T} \geq 0
$$

Où $\Gamma$ is total dissipation; $\mathrm{q}$ is the heat flux vector; $\frac{q \cdot \nabla T}{T}$ is the dissipation caused by the heat flow.

$$
Q=-k \cdot \nabla T
$$

Taking into account the Fourier law on heat flux, the result total dissipation $\Gamma$ is:

$$
\begin{gathered}
\Gamma=\left(\sigma-\frac{\partial \Psi}{\partial \varepsilon^{e}}\right): \dot{\varepsilon}^{e}+\left(-\eta-\frac{\partial \Psi}{\partial T}\right) \dot{T}+\sum_{n=1}^{24}\left[g(\sigma+B): R^{n}-\alpha\left(T-T_{0}\right)-Y^{n}-\right. \\
\left.\frac{1}{2} \varepsilon^{e}: \Delta S: \varepsilon^{e}\right] x+\sum_{n=1}^{24}\left[(\sigma+B): R^{n}\right] \dot{\gamma}^{n}+\frac{K:(\nabla T \otimes \nabla T)}{T} \geq 0
\end{gathered}
$$

From Equation (10), the elastic stress-strain relationship, the entropy-temperature equation, and the relative thermodynamic driving forces can be obtained as follows:

$$
\begin{gathered}
\sigma=\frac{\partial \Psi}{\partial \varepsilon^{e}}=S: \varepsilon^{e} \\
\eta=-\frac{\partial \Psi}{\partial T}=-\alpha x+c \ln \left(\frac{T}{T_{0}}\right) \\
F_{t r}^{n}=g(\sigma+B): R^{n}-\alpha\left(T-T_{0}\right)-Y^{n}-\frac{1}{2} \varepsilon^{e}: \Delta S: \varepsilon^{e} \\
F_{i r}^{n}=g(\sigma+B): R^{n}-\alpha\left(T-T_{0}\right)-Y^{n}-\frac{1}{2} \varepsilon^{e}: \Delta S: \varepsilon^{e} \\
F_{\gamma}^{n}=(\sigma+B): R^{n}
\end{gathered}
$$

The dissipation produced by the inelastic deformation is positive that:

$$
\Gamma_{i n t}=\sum_{n=1}^{24}\left[F_{t r}^{n} \dot{x}_{i r}^{n}+F_{i r}^{n} \dot{x}_{i r}^{n}+F_{\gamma}^{n} \dot{\gamma}^{n}\right] \geq 0
$$

Similarly, it is easy to obtain that the heat dissipation is non-negative because the tensor $\mathrm{k}$ is defined positive, that is to say:

$$
\frac{k:(\nabla T \otimes \nabla T)}{T} \geq 0
$$


Moreover, the dissipation due to the heat is non-negative, then:

$$
-\frac{1}{T} Q \cdot \nabla T \geq 0
$$

Considering the polycrystalline domain $\Phi$, which is domain, has many grains, and the number of grains is $\mathrm{n}$. The surface of the domain is denoted $S_{T}$. Integration equation (4. 37) in all the domain $\Phi$ gives:

$$
\begin{gathered}
c \frac{\partial}{\partial t} \int_{\Phi} T(x, t) d V-\int_{\Phi} \nabla \cdot(k . \nabla T) d V=\int_{\Phi} g(x, t) d V \\
g(x, t)=\sum_{n=1}^{24} F_{t r}^{n}(Y, t) x_{r e}^{n}(Y, t)+\sum_{n=1}^{24} F_{t r}^{n}(Y, t) x_{i r}^{n}(Y, t)+\sum_{n=1}^{24} F_{\gamma}^{n}(Y, t) \dot{\gamma}^{n}(Y, t)+ \\
\alpha T(Y, t) \sum_{n=1}^{24} \dot{x}^{n}(Y, t)
\end{gathered}
$$

Where, $\mathrm{g}(\mathrm{x}, \mathrm{t})$ is the internal heat source and $\mathrm{V}$ is the volume of the domain $\Phi$.

Then, the overall means temperature, and the internal heat source in the whole domain $\Phi$ can be simply defined as ([17]-[18]-[20]) :

$$
\begin{aligned}
& T(t)=\frac{1}{V} \int_{\Phi} T(x, t) d V \\
& g(x)=\frac{1}{V} \int_{\Phi} g(x, t) d V
\end{aligned}
$$

Substitution equations (4. 43) and (4.44) in equation (4.40) and remembering that volume integrals can be converted to surface integrals using Gaussian theory:

$$
C_{v} V \frac{d T}{d t} g V+\oint_{S_{T}} n(k . \nabla T) d S_{T}
$$

Where $\mathrm{n}$ is the normal direction to the surface of $S_{T}$.

Equation (4.45) describes the evolution of global average temperature in the whole field of polycrystalline materials. Considering the Newton boundary condition, that is:

$$
\text { n. }(K \Delta T)=h\left(T_{r}-T_{S}\right) \text { in } \quad S_{T}
$$

Where $T_{r}$ the ambient temperature is $T_{S}$ is the surface temperature in $S_{T}$ and h is the heat exchange coefficient of the ambient medium. It is known that the temperature field in the domain $\Phi$ is heterogeneous during the transformation of martensite due to the existence of subdomains and interfaces [21]-[22]-[23].

The temperature of each point of material in the domain $\Phi$ and the surface $S_{T}$ can be 
considered as identical to the global average temperature:

$$
\begin{gathered}
T=T_{s}=T_{m} \\
c \frac{d T}{d t}=g+\frac{h\left(T_{r}-T\right) \bar{S}}{V}
\end{gathered}
$$

As a result, taking into account the assumption of a uniform temperature field, the Equation. (4.36) is reduced to the equation (4.48) when the Newton boundary condition is assumed.

Accordingly, from the geometrical parameters, it is then possible to get the thermal parameters (radiation, heat needed for phase transition) to estimate the power required to activate the martensitic transformation. Therefore, the Shape Memory Alloy changes shape, size, or cause a dislocation by applying a significant load on a definite weight reacting to thermal stimulus that can induce the martensite transformation.

The emission of electromagnetic waves can transmit thermal radiation; radiation happens through space or any transparent intermediate (either solid or fluid). As a result, Solar radiation is also electromagnetic wave can transfer energy (E) comes from the sun; these waves characterize by the wavelength $(\lambda)$ and can transfer heat through a vacuum with the speed of light (c). This energy can be describing:

$$
E=h \cdot \frac{c}{\lambda}
$$

This solar radiation energy can be absorbed or released only in the frame of very small energy parts that can not be divided. Generally, the sunshine increases the energy quantity received from the sun at one location. Therefore, the body that receipts electromagnetic radiation can not absorb all the radiations received there is some part of these waves is reflected. The energy absorbed changes to thermal energy, which increases the body temperature. In this state, considering Boltzmann's law states that the full energy released from the black body[24] is:

$$
E(T)=e \cdot \sigma \cdot T^{4}
$$

Then, the temperature received is:

$$
T=\sqrt[4]{\frac{E}{e \sigma}}
$$

Were, $\sigma=5.66 \mathrm{e}^{-8} \mathrm{~W} / \mathrm{m}^{2} \cdot \mathrm{K}^{4}=$ Stephan-Boltzmann constant and e the emissivity correspond to the radiative flux emitted by surface element as thermal radiation. 
Consequently, the work giving by radiation heat is a major energy that can change the SMA phase, which make the wire accurate from the martensite to austenite. As long as, the SMA heated by the thermal radiation, consequently this acquired energy can be stored as an austenite shape. Since, the radiation heat is disappeared the acquired energy releases as a mechanical energy.

The sun provides an important thermal energy, which can be able to activate the martensite transformation. In this way, the SMA can react to thermal stimulus by applying mechanical force. The SMA process is stretched in the martensitic phase, below the temperature Ms after the SMA is exposed to radiation effect [25]

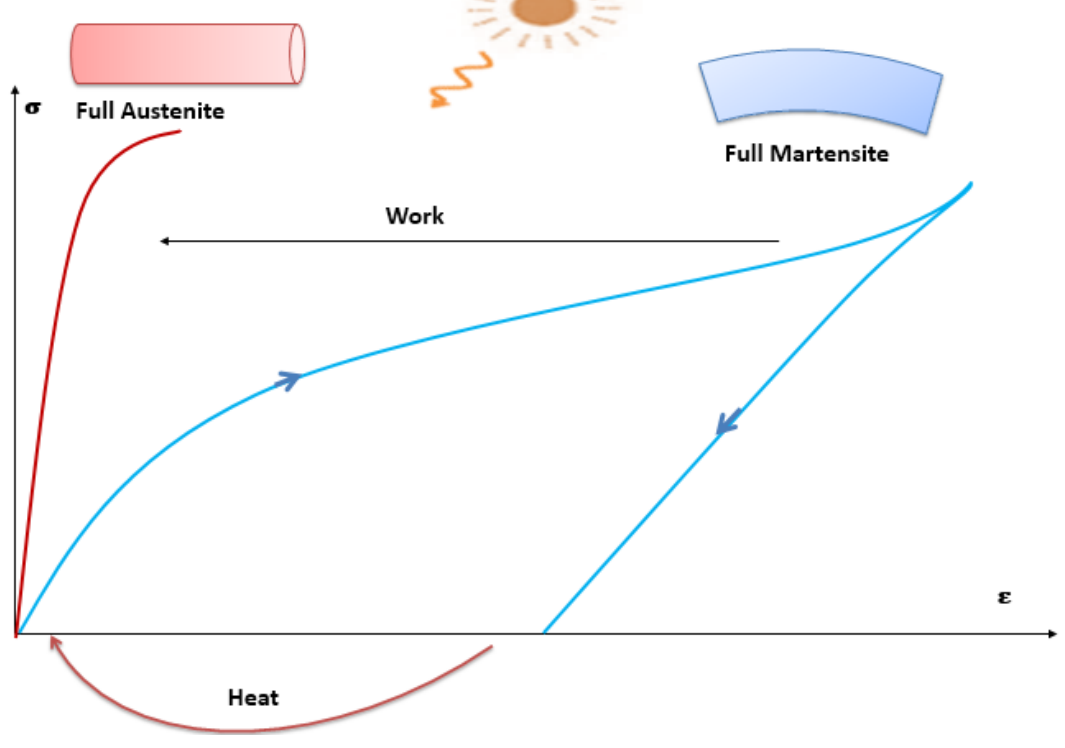

Figure 4: Work extraction from SMA.

The radiation heat is a thermal energy allows transforming the SMA tube from the martensite phase to the austenite phase, which causes mechanical work. As a result, the thermal energy stored in the SMA can be released as mechanical energy in the absence of heating[26].

Consequently, the cycling process between cooling figure 5 (c) and heating figure 5 (a) can be repetitive as presented in figure 5 . 


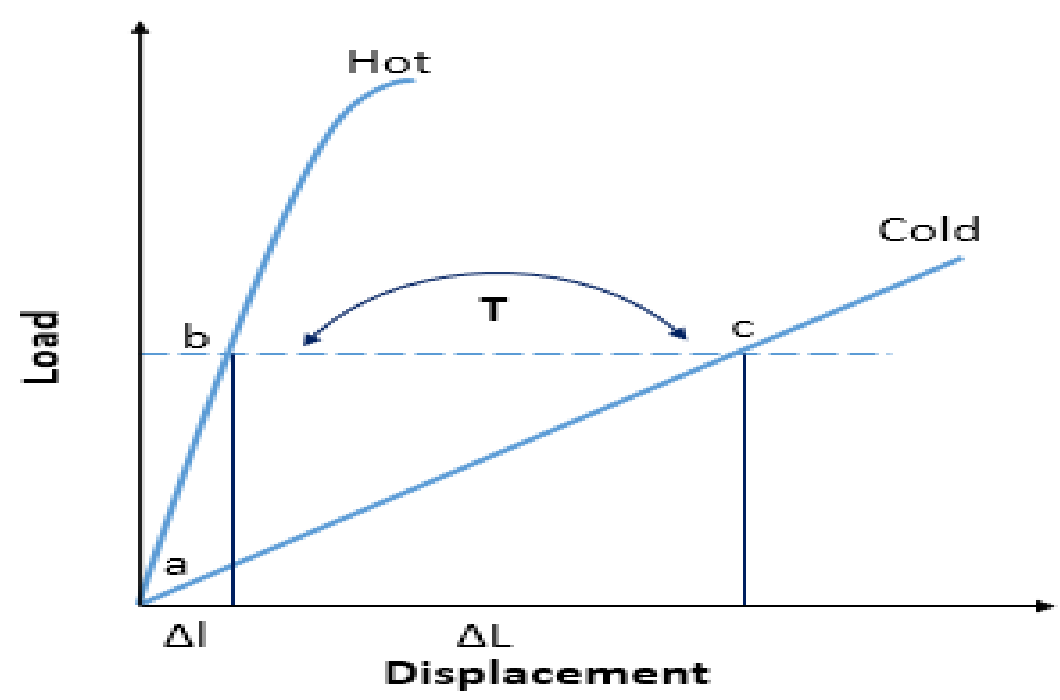

Figure 5: the curves at high and low temperatures of SMA Ni-Ti

The ability of shape memory alloy to accurate between two shapes at higher temperature and at lower temperature with displacement and lift specific weight allow this material to be good actuators and sensors. The capacity of this type of thermo-mechanical actuator depends on the kind of material used, the geometry, the weight, the rate of the sun radiation. Therefore, the shape memory alloy is an intelligent material could achieve better performance if that consider all these elements in the modelling procedure [27].

\section{RESULTS}

In order to validate the thermomecahnical model a numerical code implemented in matlab and the results are compared with the data in the literature, the material properties for mathematical simulations is cited in Table 1 [28].

Firstly, it has been considered that the temperature used for heat is equal to that one employed in the normal day's temperature. The model output has been tested for the variation of stress, temperature and strain in time. The results are compared with previous experimental results of conduction heat, hence the results are good. The obtained results are presented below: 

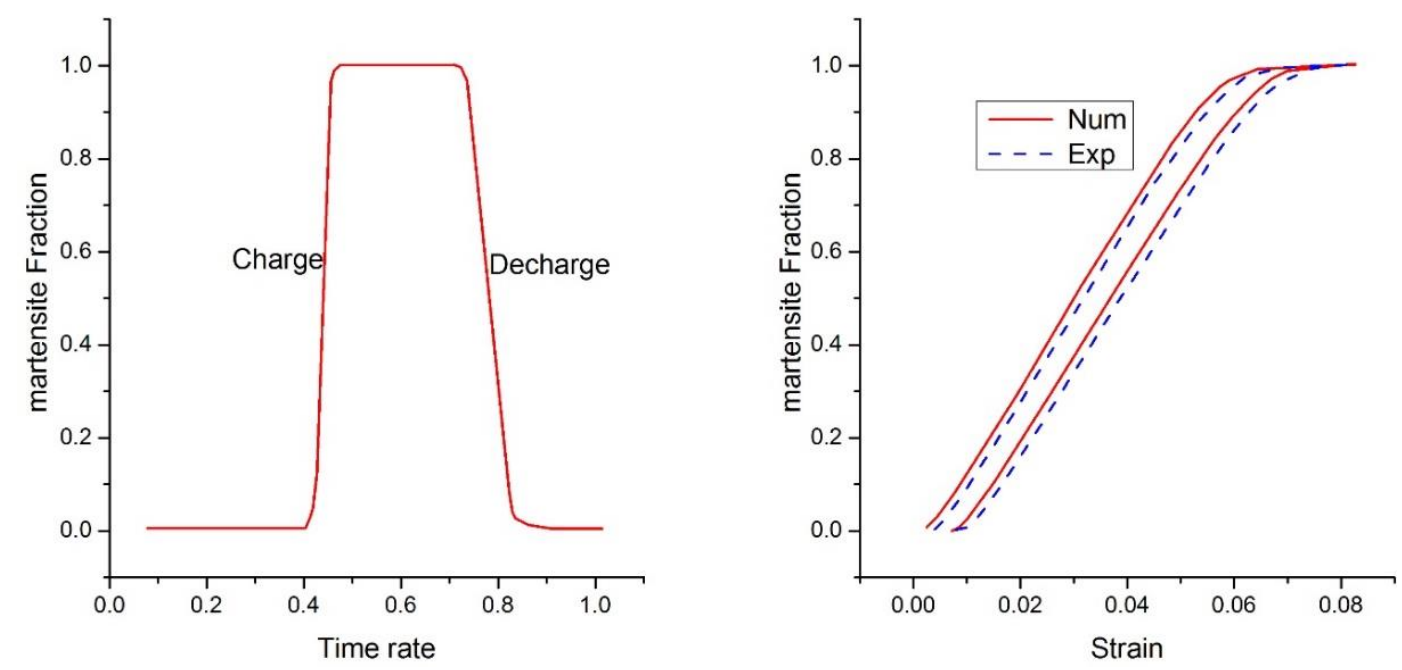

Figure 6 : a) Evolution of the martensite volume fraction. b) Associated martensite change of fraction during loading and unloading

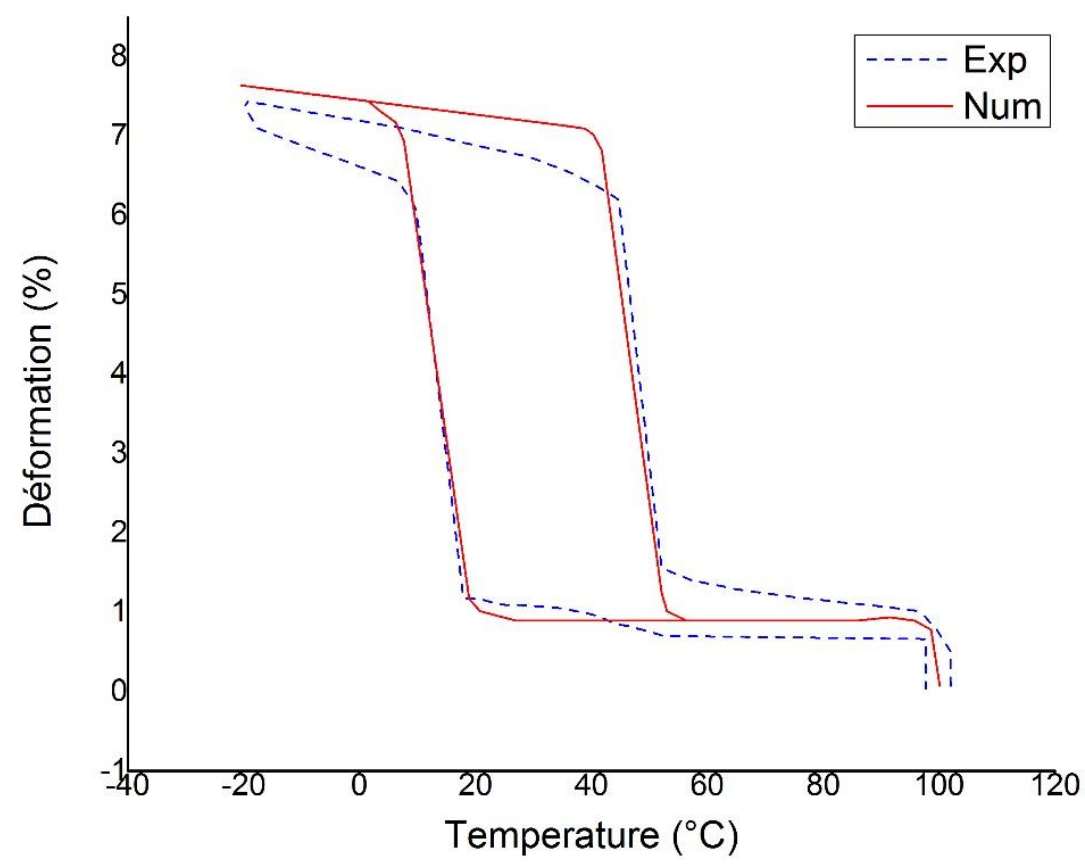

Figure 7: The Strain-Temperature curve of the SMA; the model response for a thermal cycle as a function of strain under constant stress [29]

The SMA behavior respond to radiation change as strain function and temperature. The radiation heat effect can be activated the martensite transformation conversely the absence of radiation deactivated the transformation.

As shown in figure 7 , firstly the SMA is in martensite phase below $20^{\circ} \mathrm{C}$ the strain in the maximum value, after the SMA is heated by solar radiation and the temperature increase until $40^{\circ} \mathrm{C}$ gradually in the material as the strain decrease the material return to its original shape. 
Therefore, the SMA behavior was verified in different temperature when the strain related to stress to induce the martensite transformation. As a result, the model can capture the superelasticity that is why can return to its original shape directly after deformation above $40{ }^{\circ} \mathrm{C}$. The simulation results for mechanical loading tests at different temperatures are consistent with those obtained experimentally, as shown in figure 8 and figure 9.
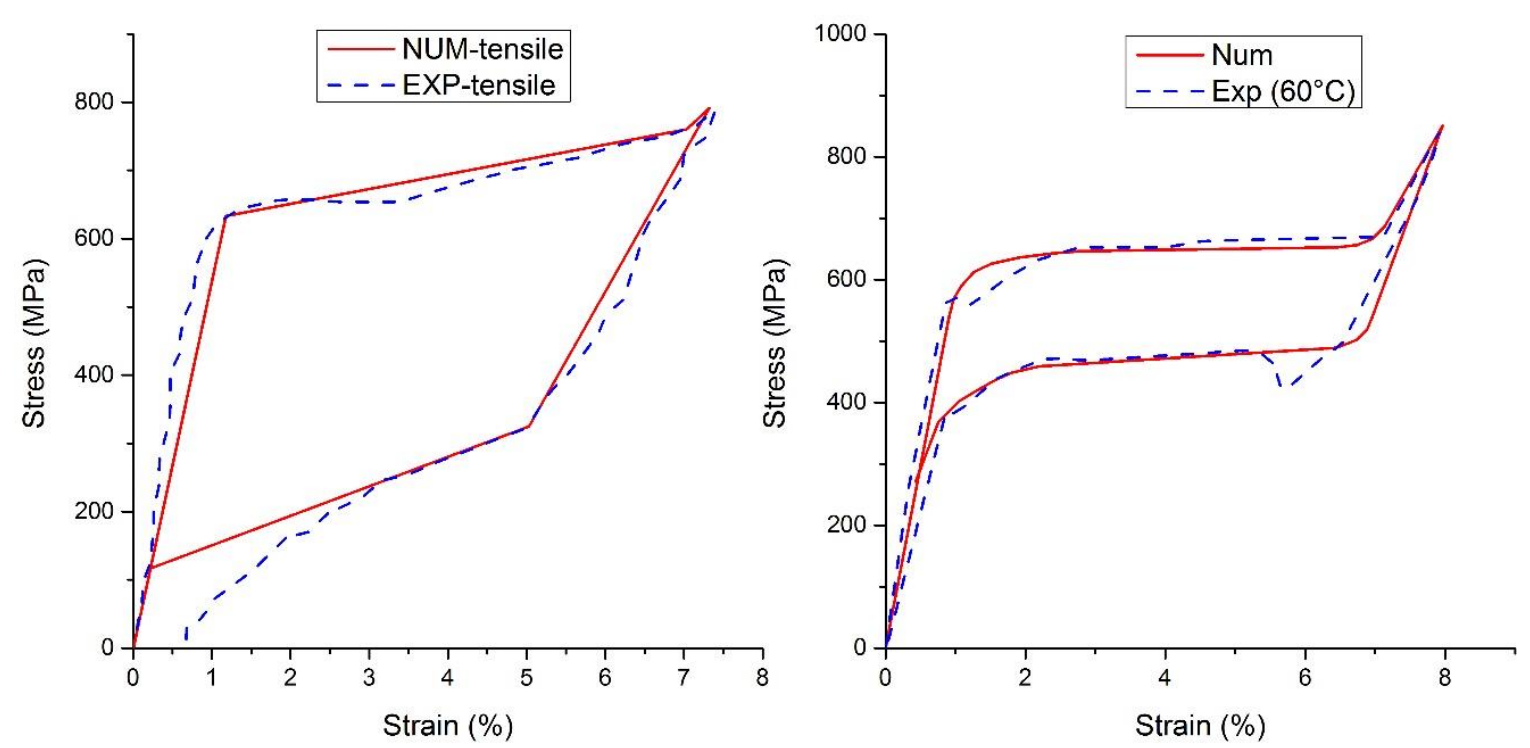

Figure 8: Superelastic prediction of the numerical model compared with experimental data for isothermal and non-isothermal tensile loading for $\mathrm{Ni}-\mathrm{Ti}$
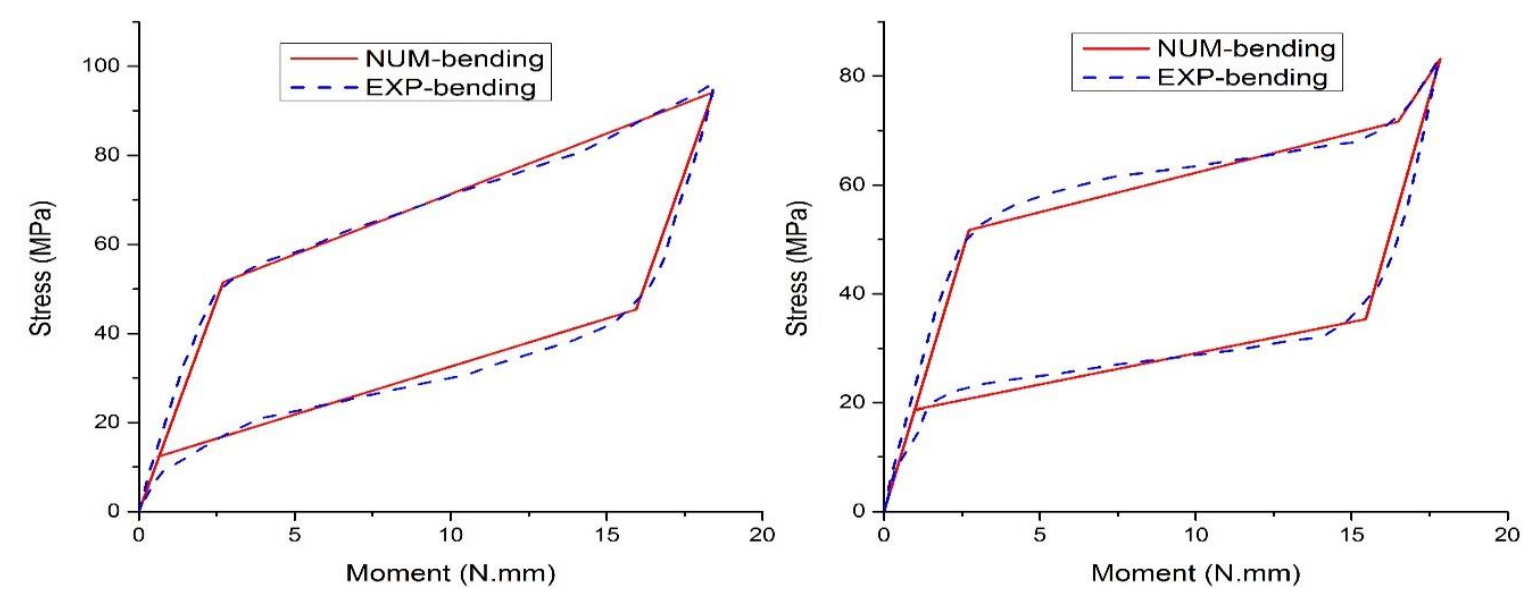

Figure 9: Superplastic prediction of the numerical model compared with experimental data for isothermal and non-isothermal bending loading for $\mathrm{Ni}-\mathrm{Ti}$

In addition, the thermomecanical model successes to capture the shape memory effect for SMA actuator at different thermomechanical condition [23][24]. As shown in figure 10 and figure 11: 

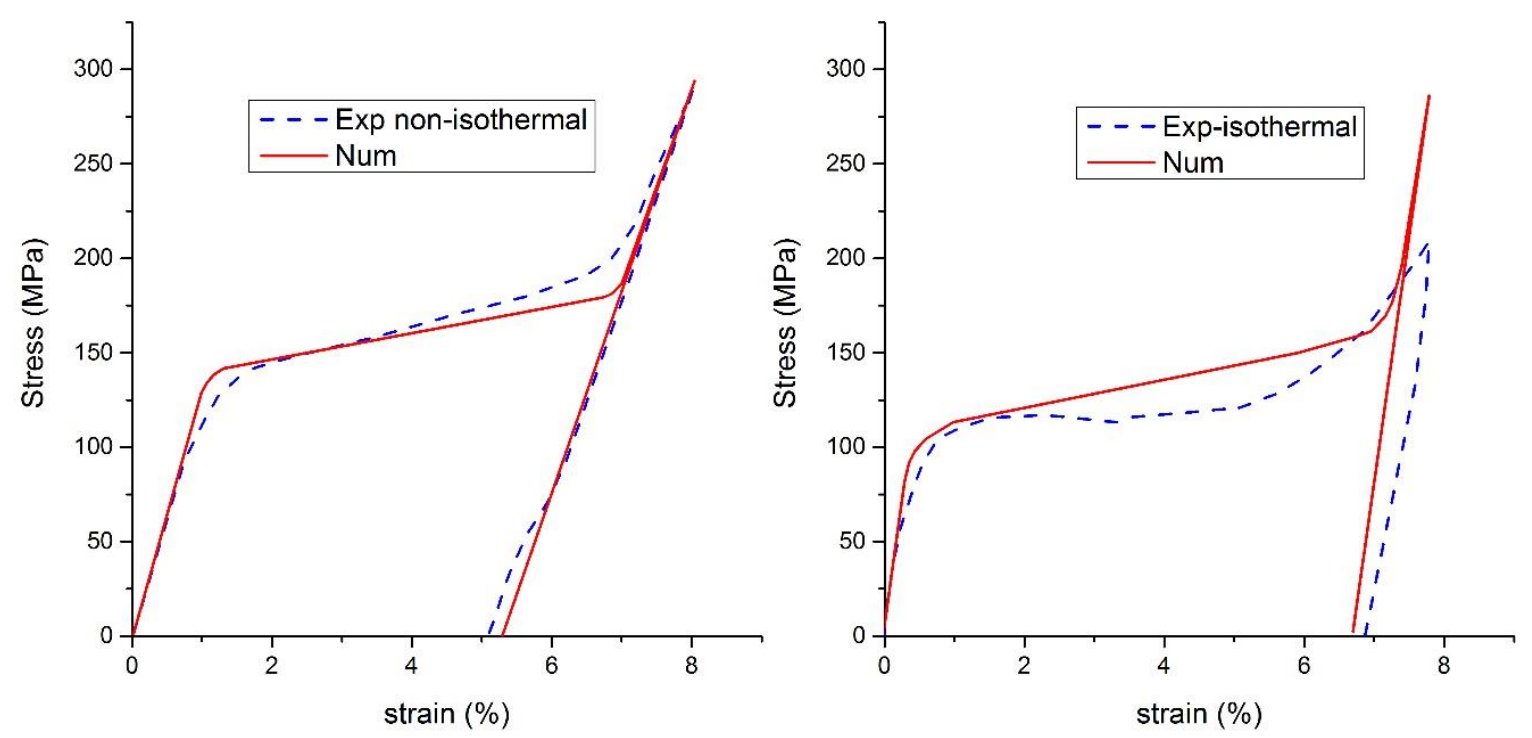

Figure 10: the prediction of the memory effect of the numerical model compared with the experimental data for non-isothermal traction for $\mathrm{Ni}-\mathrm{Ti}$, a) isothermal b) non-isothermal
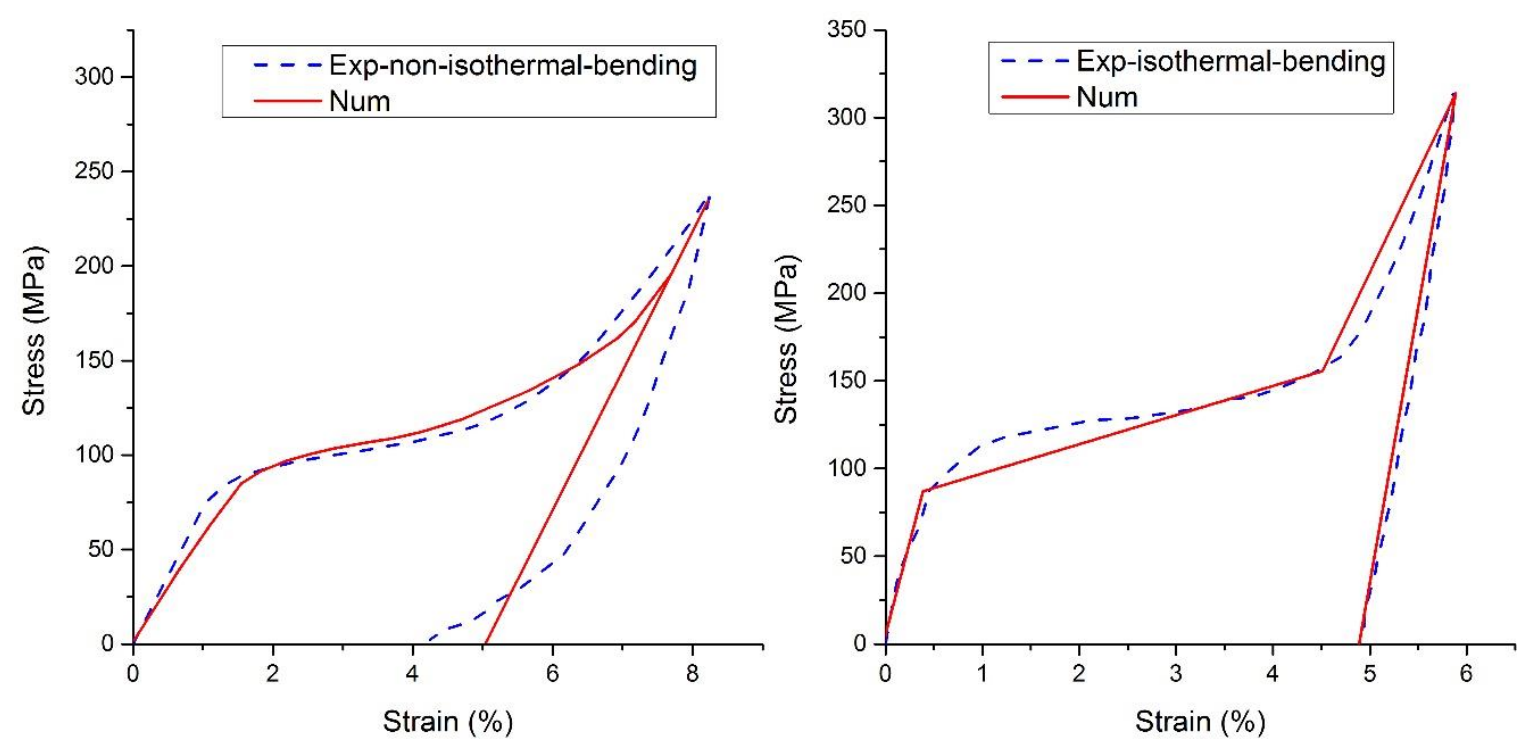

Figure 31: the prediction of the memory effect of the numerical model compared with the experimental data for non-isothermal bending for $\mathrm{Ni}-\mathrm{Ti}$, a) isothermal b) nonisothermal

The shape memory effect is established and the material exhibits elastic behavior while the load remains below the critical stress, after self-accommodated martensite turns into oriented martensite. The unloading results only an elastic recovery, leaving a residual stress. To complete the shape memory effect, the material is heated to a temperature above the temperature by the end of the austenite at zero force; during this stage, residual deformation is restored [29]. 
It can be deduced that the shape memory effect and the superelasticity are connected to the thermo-mechanical loading. Therefore, the SMA require superelastic behavior under $A_{f}$, when the temperature decreased below $A_{f}$, the SMA require shape memory effect behavior as shown in figure 12:
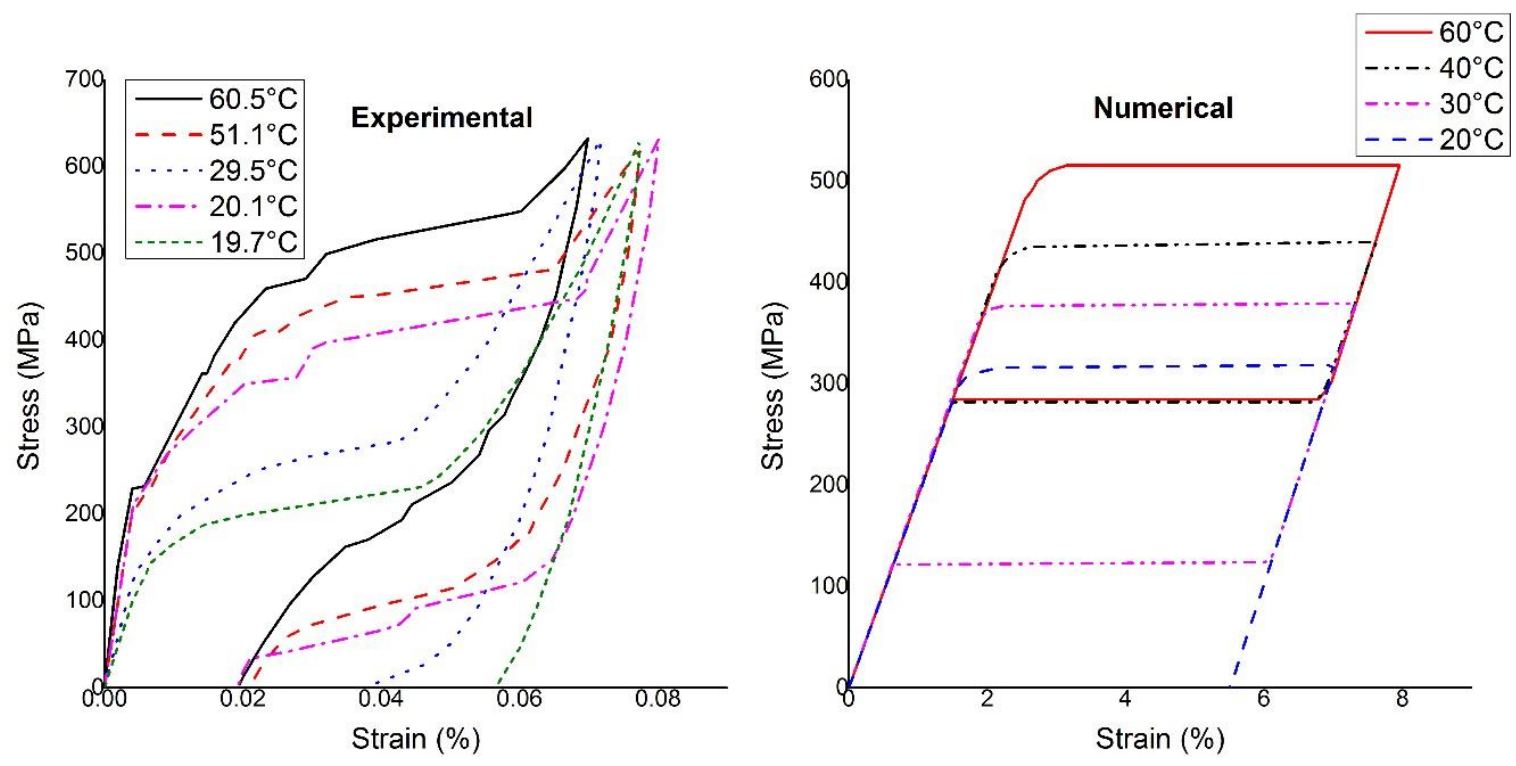

Figure 12: simulation at different temperatures

The following simulation was performed on Ni-Ti SMA with a non-proportional loading path in the form of a butterfly-shaped strain was considered qualitatively, note that each of the computed stress paths due to a path of giving constraint closely corresponds to the experimental observations reported in the literature [31] as presented in figure 13:
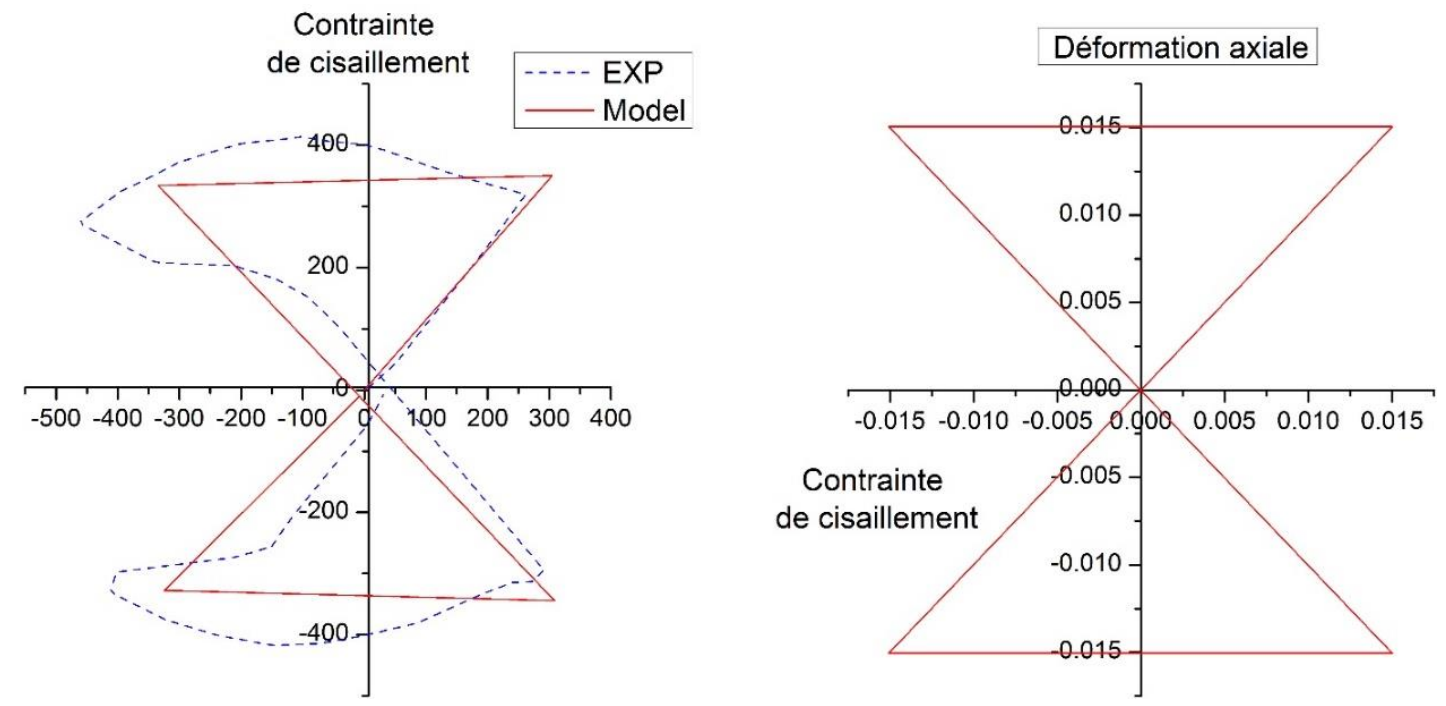

Figure 4: Comparison between the numerical and experimental results of butterfly type non-proportional multiaxial loading paths 
To demonstrate the influence of thermomechanical coupling on shape memory alloy behavior, the pseudoelastic simulations for uniaxial tensile tests under non-isothermal boundary conditions are performed in figure 14 .

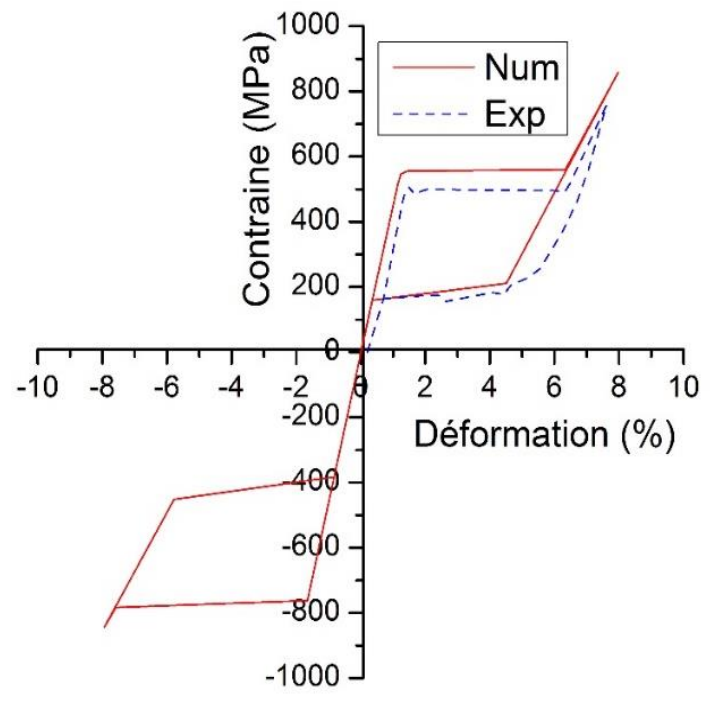

(a)

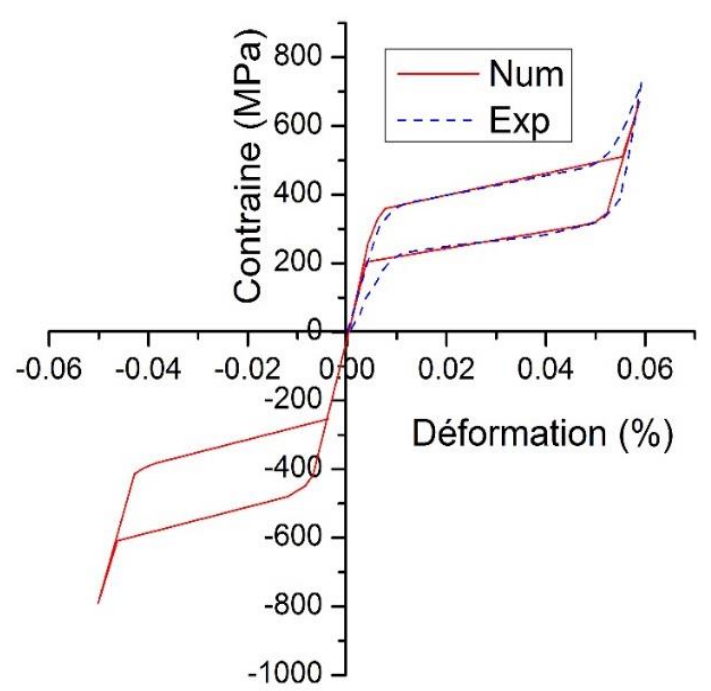

(b)

Figure 5: Tensile and compressive loadings for different levels strain of dissymmetry behavior and internal loops [32]

The comparison of the numerical and experimental results shows, on the one hand, that the model used accurately describe the respective SMA behaviors, and on the other hand that the modeling correctly reproduces the effects of structural interactions between the constituents.

\section{Conclusion}

The radiation effect can be a significant way more efficient than the other method of heating that can replace conduction and convection to benefit from the intelligent behavior of the shape memory alloy in all the application have been existed also the renewable and industrial application[21]. It was the main purpose of the investigation to get attention to the shape memory alloys heated by thermal radiation specially the thermal radiation of the sun.

Therefore, a numerical model aims to describe the principal thermomecanical behavior for shape memory alloy has been proposed. As a result, the simulation in MATLAB was used to study both the shape memory effect and superelasticity for SMA features in different thermal and mechanical conditions. 
Consequently, this feature of shape memory alloys is very interesting and it can be used in many applications (industry, renewable energy and microsystems...). It can offer large strain react to radiation effect comes from the sun, which gives opportunities to design many actuators and sensors for clean energy [33].

\section{References}

[1] T. L. Grigorie, A. V. Popov, R. M. Botez, M. Mamou, and Y. Mébarki, "On-off and proportional-integral controller for a morphing wing. Part 1: Actuation mechanism and control design," Proc. Inst. Mech. Eng. Part G J. Aerosp. Eng., vol. 226, no. 2, pp. 131-145, 2012.

[2] A. Riad, M. Ben Zohra, M. Mansouri, and A. Alhamany, "The shape memory alloy controlled by the sun's radiation effect," J. Comput. Methods Sci. Eng., vol. 18, no. 1, pp. 117-127, Feb. 2018.

[3] H. Tobushi, T. Hachisuka, T. Hashimoto, and S. Yamada, "Cyclic Deformation and Fatigue of a TiNi Shape-Memory Alloy Wire Subjected to Rotating Bending," J. Eng. Mater. Technol., vol. 120, no. January 1998, pp. 64-70, 1998.

[4] M. W. M. Van Der Wijst, "Shape Memory Alloys Featuring Nitinol," TU Eindhoven, p. $62,1992$.

[5] J. Wang, Z. Moumni, W. Zhang, and W. Zaki, "A thermomechanically coupled finite deformation constitutive model for shape memory alloys based on Hencky strain," Int. J. Eng. Sci., vol. 117, pp. 51-77, 2017.

[6] A. Alhamany, M. O. Bensalah, and O. F. Fehri, "Couplage dans les alliages ?? m??moire de forme," Comptes Rendus - Mec., vol. 332, no. 11, pp. 941-947, 2004.

[7] P. Sittner, Y. Hara, and M. Tokuda, "Experimental study on the thermoelastic martensitic transformation in shape memory alloy polycrystal induced by combined external forces," Metall. Mater. Trans. A, vol. 26, no. 11, pp. 2923-2935, 1995.

[8] G. Yun and H. S. Park, "A multiscale, finite deformation formulation for surface stress effects on the coupled thermomechanical behavior of nanomaterials," Comput. Methods Appl. Mech. Eng., vol. 197, no. 41-42, pp. 3337-3350, 2008.

[9] K. Guda Vishnu and A. Strachan, "Phase stability and transformations in NiTi from density functional theory calculations," Acta Mater., vol. 58, no. 3, pp. 745-752, 2010. 
[10] J. A. Shaw, "Simulations of localized thermo-mechanical behavior in a NiTi shape memory alloy," Int. J. Plast., vol. 16, pp. 541-562, 2000.

[11] A. Riad, A. Alhamany, and M. Benzohra, "The shape memory alloy actuator controlled by the Sun's radiation," Mater. Res. Express, vol. 4, no. 7, p. 075701, Jul. 2017.

[12] R. Velázquez and E. E. Pissaloux, "Modelling and temperature control of shape memory alloys with fast electrical heating," Int. J. Mech. Control, vol. 13, no. 2, pp. 3-10, 2012.

[13] H. Ma, "Thermal Modeling of Shape Memory Alloy Wire Actuators for Automotive Applications," 2010.

[14] J. Bartl and M. Baranek, "Emissivity of aluminium and its importance for radiometric measurement," 2004.

[15] D. Entemeyer, "Etude micromécanique du comportement thermomécanique des alliages à mémoire de forme To cite this version : HAL Id : tel-01777144 soutenance et mis à disposition de 1 , ensemble de la Contact : ddoc-theses-contact@univlorraine.fr," 2018.

[16] D. C. Lagoudas, P. B. Entchev, P. Popov, E. Patoor, L. C. Brinson, and X. Gao, "Shape memory alloys, Part II: Modeling of polycrystals," Mech. Mater., vol. 38, no. 5-6, pp. 430-462, 2006.

[17] Y. J. He and Q. P. Sun, "International Journal of Solids and Structures On nonmonotonic rate dependence of stress hysteresis of superelastic shape memory alloy bars," vol. 48, pp. 1688-1695, 2011.

[18] H. Yin and Q. Sun, "Temperature variation in niti shape memory alloy during cyclic phase transition," J. Mater. Eng. Perform., vol. 21, no. 12, pp. 2505-2508, 2012.

[19] H. Yin, Y. Yan, Y. Huo, and Q. Sun, "Rate dependent damping of single crystal CuAlNi shape memory alloy," Mater. Lett., vol. 109, pp. 287-290, 2013.

[20] H. Yin, Y. He, and Q. Sun, "Journal of the Mechanics and Physics of Solids Effect of deformation frequency on temperature and stress oscillations in cyclic phase transition of NiTi shape memory alloy,” J. Mech. Phys. Solids, vol. 67, pp. 100-128, 2014.

[21] J. Shaw, "Thermomechanical aspects of NiTi," J. Mech. Phys. Solids, vol. 43, no. 8, pp. 1243-1281, Aug. 1995.

[22] Q. P. Sun and Z. Q. Li, "Phase transformation in superelastic NiTi polycrystalline 
micro-tubes under tension and torsion - From localization to homogeneous deformation,” Int. J. Solids Struct., vol. 39, no. 13-14, pp. 3797-3809, 2002.

[23] X. Zhang, P. Feng, Y. He, T. Yu, and Q. Sun, "Experimental study on rate dependence of macroscopic domain and stress hysteresis in NiTi shape memory alloy strips,” Int. J. Mech. Sci., vol. 52, no. 12, pp. 1660-1670, 2010.

[24] C. Johnson, "Mathematical Physics of BlackBody Radiation," 2012.

[25] A. Riad, A. Alhamany, and M. Benzohra, "The shape memory alloy actuator controlled by the Sun's radiation," Mater. Res. Express, 2017.

[26] N. J. Bechle and S. Kyriakides, "Localization in NiTi tubes under bending," Int. J. Solids Struct., vol. 51, no. 5, pp. 967-980, 2014.

[27] M. Tabesh and M. H. Elahinia, "\&lt;title\&gt;Modeling and optimization of shape memory-superelastic antagonistic beam assembly\&lt;/title\&gt;", vol. 7643, p. 76430A-76430A-12, 2010.

[28] N. J. Ganesh, S. Maniprakash, L. Chandrasekaran, S. M. Srinivasan, and A. R. Srinivasa, "Design and Development of a Sun Tracking Mechanism Using the Direct SMA Actuation," J. Mech. Des., vol. 133, no. 7, p. 075001, 2011.

[29] F. Auricchio, E. Bonetti, G. Scalet, and F. Ubertini, "Theoretical and numerical modeling of shape memory alloys accounting for multiple phase transformations and martensite reorientation," Int. J. Plast., vol. 59, pp. 30-54, 2014.

[30] Z. Karbaschi, "Torsional behavior of nitinol: modeling and experimental evaluation," 2012.

[31] D. Helm, "Pseudoelastic Behavior of Shape Memory Alloys: Constitutive Theory and Identification of the Material Parameters Using Neural Networks *," Tech. Mech., no. 1, pp. 39-58, 2005.

[32] Y. Chemisky, "Modelisation macroscopique des alliages a memoire de forme Application aux materiaux composites," Paul Verlaine de Metz, 2009.

[33] A. Riad, M. Benzohra, M. Mansouri, and A. Alhamany, "Design of Sunflower System Based on Shape Memory Alloy Actuator,” pp. 45-54, 2018. 